\title{
SWEEPING OUT ON A SET OF INTEGERS
}

\author{
MARTIN H. ELLIS AND NATHANIEL A. FRIEDMAN ${ }^{1}$
}

\begin{abstract}
Let $(X, \mathscr{B}, m)$ be a Lebesgue space, $m(X)=1$, and let $T$ be an invertible measurable nonsingular aperiodic transformation of $X$ onto $X$. If $S$ is a set of $r$ integers, $r \geqslant 2$, then there exists a set $A$ of measure less than $r^{-1} \sum_{k=1}^{r} k^{-1}$ such that $X=\bigcup_{n \in S} T^{n} A$. Thus for every infinite set of integers $W$ there exist sets $A$ of arbitrarily small measure such that $X=$ $\bigcup_{n \cap W} T^{n} A$.
\end{abstract}

1. Introduction. Let $(X, \mathscr{B}, m)$ be a Lebesgue space, $m(X)=1$, and let $\mathcal{T}$ denote the class of invertible measurable nonsingular aperiodic transformations $T$ mapping $X$ onto $X$. T is measurable if images of measurable sets under $T$ and $T^{-1}$ are measurable and $T$ is nonsingular if images of sets of measure zero under $T$ and $T^{-1}$ have measure zero. $T$ is aperiodic if the set of points $x$ such that $T^{n} x=x$ has measure zero for each $n \geqslant 1$. Hereafter all transformations considered are assumed to be in $\mathcal{T}$.

A transformation $T$ is measure preserving if images of a measurable set under $T$ and $T^{-1}$ have the same measure as the set. A transformation $T$ is ergodic if $T A=A$ implies $m(A)=0$ or 1 . An ergodic transformation is aperiodic since $m$ is nonatomic. $T$ is mixing if

$$
\lim _{n \rightarrow \infty} m\left(T^{n} A \cap B\right)=m(A) m(B), \quad A, B \in \mathscr{B} .
$$

If $T$ is mixing, then $T$ is ergodic and measure preserving.

Let $S$ be a finite or infinite set of integers. If $1=m\left(\cup_{n \in S} T^{n} A\right)$, then we say that $A$ sweeps out on $S$. If $T$ is mixing (or just partially mixing [6]), $S$ is infinite, and $m(A)>0$, then it is not difficult to show that $A$ sweeps out on $S$. In particular, there exist sets of arbitrarily small measure that sweep out on $S$.

In order to show that for each transformation in $\sigma$ there exist sweep out sets of arbitrarily small positive measure on every infinite set of integers, we shall study the question of how small can the measure of a set be if the set sweeps out on a finite set of integers. Let $S=\left\{n_{1}<n_{2}<\cdots<n_{r}\right\}$ be a set of $r$ integers, where $r \geqslant 2$, and let

$$
g(S, T)=\inf \left\{m(A): \bigcup_{i=1}^{r} T^{n_{i}} A=X\right\} .
$$

It will be shown that $g(S, T)<r^{-1} \sum_{i=1}^{r} k^{-1}$. To prove this result we shall

Received by the editors February 22, 1978.

AMS (MOS) subject classifications (1970). Primary 28A65; Secondary 54H20.

${ }^{1}$ The research of the first author was partially supported by National Science Foundation Grant MCS7703659, research of the second author by MCS7606735A01. 
use the following theorem which is due to Rohlin [7] in the measure preserving case. It was proved in [1] in the nonsingular case and a general discussion of this case is given in $[5, \S 7]$.

THEOREM 1.3. Given $T \in \mathcal{T}$, a positive integer $r$, and $\varepsilon>0$, there exists $B \in \mathscr{B}$ such that $T^{i} B, 0 \leqslant i<r$, are disjoint and $m\left(\cup_{i=0}^{r-1} T^{i} B\right)>1-\varepsilon$.

Note that if $S=\{0,1,2, \ldots, r-1\}$ then Theorem 1.3 implies $g(S, T) \leqslant$ $1 / r$, with equality if $T$ is measure preserving.

2. Preliminaries. All iterates $T^{i}$ are nonsingular since $T$ is nonsingular. Thus each measure $m\left(T^{i}\right)$ is absolutely continuous with respect to $m$, which implies the following result.

LEMMA 2.1. Let $t$ be a positive integer. For each $\varepsilon>0$ there exists $\delta=\delta(\varepsilon, t)$ $>0$ such that $m(A)<\delta$ implies $m\left(\cup_{i=-t}^{t} T^{i} A\right)<\varepsilon$.

Given a set of integers $D$, let $|D|$ denote the cardinality of $D$. Given a positive integer $h$ and sets of integers $D$ and $E$, let

$(D+E) \bmod h$

$$
=\{u: 0 \leqslant u \leqslant h-1, u=(d+e) \bmod h \text { for some } d \in D, e \in E\} .
$$

Hereafter $h$ will always be a positive integer and $H$ will denote $\{0,1,2, \ldots, h-1\}$.

LEMMA 2.2. Let $S=\left\{n_{1}, \ldots, n_{r}\right\}$ be a set of $r$ integers and let $h>n_{r}-n_{1}$. Let $D \subset H$ and $|D|>k h / r$, where $k \in\{0,1,2, \ldots, r-1\}$. Then there exists $p \in H$ such that

$$
|((\{p\}+S) \bmod h) \cap D| \geqslant k+1
$$

Proof. For $m \in\{0,1,2, \ldots, r\}$ let

$$
C_{m}=\{x \in H:|((\{x\}+S) \bmod h) \cap D|=m\} \text {. }
$$

Since $h>n_{r}-n_{1}$,

$$
\sum_{m=0}^{r} m\left|C_{m}\right|=r|D|>k h .
$$

Inequality (2) implies $\left|C_{j}\right| \geqslant 1$ for some $j \geqslant k+1$.

LEMMA 2.3. Given $S$ and $h>n_{r}-n_{1}$, there exists $E \subset H$ such that $(E+$ $S) \bmod h=H$ and $|E| \leqslant r^{-1} h \sum_{k=1}^{r} k^{-1}$.

Proof. Let $p_{1}=0$. If $p_{1}, p_{2}, \ldots, p_{j}$ have been defined and

$$
h_{j}=\left|\left(\left\{p_{1}, p_{2}, \ldots, p_{j}\right\}+S\right) \bmod h\right|
$$

satisfies $h_{j}<h$, then choose $p_{j+1} \in H$ so that $h_{j+1}$ is maximal. Let $v$ be the positive integer such that $h_{v}=h$ and let $E=\left\{p_{1}, p_{2}, \ldots, p_{v}\right\}$.

Let $n_{1}=h_{1}$ and let $n_{j}=h_{j}-h_{j-1}, 2 \leqslant j \leqslant v$. Lemma 2.2 implies $n_{j}=r$ for all $j \leqslant r h / r^{27}$, since 


$$
h-\frac{h}{r^{2}} r=\frac{h}{r}(r-1) .
$$

In general, let $u_{k}=r^{-1} h \sum_{i=0}^{k-1}(r-i)^{-1}$. Lemma 2.2 implies that for $1<k$ $<r$, if $v>j>u_{k}$, then $h_{j}>k r^{-1} h$; hence $v \leqslant u_{r}$.

3. Main result. Theorem 1.3, Lemma 2.1 and Lemma 2.3 will now be used to prove

TheOREM 3.1. If $T \in \mathcal{T}$, then $g(S, T)<r^{-1} \sum_{k=1}^{r} k^{-1}$.

Proof. Choose $h>\left|n_{1}\right|+\left|n_{r}\right|$ such that $a=r^{-1} h \sum_{k=1}^{r} k^{-1}$ is not an integer. Let $v$ be the integral part of $a$ and let $\varepsilon=a-v$. By Lemma 2.1 choose $\delta$ so that

$$
m(A)<\delta \text { implies } m\left(\bigcup_{i=-2 h}^{2 h} T^{i} A\right)<\varepsilon / h .
$$

By Theorem 1.3 there exists a measurable set $B$ such that $T^{i} B, 0 \leqslant i \leqslant h-$ 1 , are disjoint and

$$
m\left(\bigcup_{i=0}^{h-1} T^{i} B\right)>1-\delta .
$$

Let $Y=X-\cup_{i=0}^{h-1} T^{i} B$. By Lemma 2.3 there exists $E \subset H$ such that $|E|<v$ and $(E+S) \bmod h=H$. For $j \in H$ let

$$
C_{j}=\bigcup_{i \in((\{j\}+E) \bmod h)} T^{i} B
$$

Since

$$
\sum_{j=0}^{h-1} m\left(C_{j}\right)=|E| m\left(\bigcup_{i=0}^{h-1} T^{i} B\right) \leqslant|E| \leqslant v,
$$

we can fix $j$ such that $m\left(C_{j}\right) \leqslant v / h$.

Let $A=C_{j} \cup\left(\cup_{i=-2 h}^{2 h} T^{i} Y\right)$. Since $h>\left|n_{1}\right|+\left|n_{r}\right|$, we have

$$
X=\left(\bigcup_{n \in S} T^{n} C_{j}\right) \cup\left(\bigcup_{i=-h}^{h} T^{i} Y\right)
$$

hence $X=\cup_{n \in S} T^{n} A$. Lastly, (1) and (2) imply

$$
m(A) \leqslant m\left(C_{j}\right)+m\left(\bigcup_{i=-2 h}^{2 h} T^{i} Y\right)<v / h+\varepsilon / h=a / h .
$$

Thus the theorem is proven.

COROllary 3.2. If $T \in \mathcal{T}$, then for every infinite set of integers $W$ there exist sets of arbitrarily small positive measure that sweep out on $W$.

Proof. Let $\varepsilon>0$. Choose $r \geqslant 2$ so that $r^{-1} \sum_{k=1}^{r} k^{-1}<\varepsilon$. Theorem 3.1 
guarantees that for every subset $S \subset W$ which contains $r$ integers there exists a set $A$ with $m(A)<\varepsilon$ and $A$ sweeps out on $S$.

Note that if $T$ is invertible, measurable, and nonsingular but not aperiodic, then the conclusion of Corollary 3.2 cannot hold for $T$. In this case there exists a set $B$ of positive measure $q$ and a positive integer $p$ such that for all $x \in B, x=T^{p} x$. By Lemma 2.1 there exists $\delta>0$ such that $m(A)<\delta$ implies $m\left(\cup_{i=0}^{p-1} T^{i} A\right)<q$. If $X=\cup_{i=-\infty}^{\infty} T^{i} C$, then $B$ must be contained in $\cup_{i=0}^{p-1} T^{i} C$. Hence $m\left(\cup_{i=0}^{p-1} T^{i} C\right) \geqslant q$, so $m(C)>\delta$.

REMARKS. If $T_{1}$ and $T_{2}$ are measure preserving, it is not hard to show that $g\left(S, T_{1}\right)=g\left(S, T_{2}\right)$; hence $g(S, T)$ is a function $g(S)$ of $S$ in this case. In general $g(S, T) \leqslant g(S)$ for $T \in \mathcal{T}$.

In [3] Corollary 3.2 is applied to prove that for each infinite set of integers $W$ and $T \in \mathcal{T}$ there exists a countable partition that generates on $W$. In [4] Corollary 3.2 is applied to prove that for each ergodic measure-preserving translation $T$ on a compact abelian group and for each infinite set of integers $W$ there exist sets $A$ of arbitrarily small positive measure such that $\left(A, A^{c}\right)$ generates on $W$.

ADDENDUM. In [2] the following generalization of Corollary 3.2 is proved. Let $\left\{T_{i}: i \in I\right\}$ be a countable collection of invertible measurable nonsingular transformations on $X$ (transformations with periodic components allowed). There exist sets $A$ of arbitrarily small positive measure for which $X=\cup_{i \in I} T_{i} A$ if and only if

$$
m\left\{x:\left\{T_{i}^{-1}(x): i \in I\right\} \text { is finite }\right\}=0 .
$$

\section{REFERENCES}

1. R. V. Chacon and N. A. Friedman, Approximation and invariant measures, Z. Wahrscheinlichkeitstheorie und Verw. Gebiete 3 (1965), 286-295.

2. M. H. Ellis, Sweeping out under a collection of transformations (submitted).

3. M. H. Ellis and N. A. Friedman, Subset generators for nonsingular transformations (submitted).

4. Subsequence generators for ergodic group translations, Israel J. Math. (to appear).

5. N. A. Friedman, Introduction to ergodic theory, Van Nostrand-Reinhold, New York, 1969.

6. N. A. Friedman and D. S. Ornstein, On partially mixing transformations, Illinois J. Math. 16 (1972), 61-68.

7. V. A. Rohlin, In general a measure preserving transformation is not mixing, Dokl. Akad. Nauk SSSR 60 (1948), 349-351.

Department of Mathematics, State University of New York at Albany, Albany, New YoRK 12222 (Current address of N. A. Friedman)

Current address (M. H. Ellis): Department of Mathematics, Northeastern University, Boston, Massachusetts 02115 